\title{
An Analysis of the Catastrophe Model and Catastrophe Characteristics of Traffic Flow Based on Cusp Catastrophe Theory
}

\author{
Yanguo Huang $\mathbb{D}^{1}{ }^{1}$ Huimin Zhang, $^{2}$ Hongjun Liu, ${ }^{1}$ and Shengsheng Zhang ${ }^{1}$ \\ ${ }^{1}$ School of Electrical Engineering and Automation, Jiangxi University of Science \& Technology, Ganzhou 341000, China \\ ${ }^{2}$ School of Continuing Education and Training, Jiangxi University of Science \& Technology, Ganzhou 341000, China \\ Correspondence should be addressed to Yanguo Huang; jxhuangyg@126.com
}

Received 19 May 2021; Revised 25 November 2021; Accepted 10 December 2021; Published 5 January 2022

Academic Editor: Alessandro Severino

Copyright ( $(2022$ Yanguo Huang et al. This is an open access article distributed under the Creative Commons Attribution License, which permits unrestricted use, distribution, and reproduction in any medium, provided the original work is properly cited.

- The state of urban road traffic flow shows discontinuity and jumping phenomenon in the process of running. There was a data gap in the collected traffic flow data. Through the data analysis, it was found that the traffic flow state had the characteristics of multimode, mutation, inaccessibility, divergence and hysteresis, which were similar to the mutation characteristics of the basic model of catastrophe theory when the system state changed. The cusp catastrophe model of traffic flow based on traffic wave theory was established by analyzing the movement process of traffic flow. In this model, the traffic density was taken as the state variable, and traffic flow and wave speed were taken as the control variable. Referring to the basic idea of catastrophe theory, the solution method of the model was given, and the structural stability of the traffic flow state was analyzed. Through the critical equilibrium surface equation, the stability of the extreme value of the system potential function can be analyzed, and the bifurcation set equation when the traffic flow state changed can be obtained, which can be used to determine the critical range of the structural stability of the system. This paper discussed and analyzed the changing trend and constraint relationship among the wave speed, traffic density and traffic flow when the traffic flow state changed suddenly in different running environments. The analysis results were consistent with the actual road traffic flow state. A case was given, and the results showed that the cusp catastrophe model could describe the relationship among the three parameters of traffic flow from three-dimensional space, and could effectively analyze the internal relationship of the parameters when the traffic flow state changed. The validity of the model and analysis method was verified. The goal of this paper is to provide an analysis method for the judgment of urban road traffic state.

\section{Introduction}

The parameters reflecting urban road traffic flow mainly include flow, density, and speed. In the 1930s and 1940s, researchers mainly studied the traffic characteristics of free flow [1]. In this state, the traffic density was low, the headway between vehicles was large, and the vehicles were in a free driving state. Probability theory and mathematical statistics were usually used to establish traffic models. Classical models included Greenshields' linear model, Edie logarithmic model, Underwood's exponential model, and Keller's curve family model [1]. Since the 1950s, due to the increasingly weak independence of various vehicles in traffic flow, the research of traffic flow had entered the stage of nonfree flow [2]. In this state, the traffic flow density was high, the spacing between vehicles was very small, and the driving of vehicles was affected and limited by the first vehicle. A micro model based on car following theory (such as vehicle following model) and a macro model based on fluid dynamics theory were established. In 1955, Lighthill and Whitham proposed the first macroscopic traffic flow model based on fluid dynamics and proved the existence of traffic waves [3], which is also the most widely used traffic flow model so far. These traditional traffic flow theoretical models still occupy a dominant position in the current traffic flow theoretical system and have a wide range of applications.

Through the observation of actual traffic flow data, in the traffic flow parameter relationship diagram, it can often be seen that there is little or no data in the area near the traffic capacity. We called this area data gap. This gap was 
generated during the transition of two traffic states (congested state and unblocked state). It can be seen from the usual relationship diagram that there was a state jumping during the transition between states. For this phenomenon, the traditional theoretical model cannot explain the mechanism of state mutation [2]. The traditional methods based on probability theory and mathematical statistics are mostly suitable for analyzing the traffic flow parameter relationship of free flow state. For example, Greenshields' linear model is suitable for describing the state of free flow with small flow, the exponential model is more suitable for the situation with low density, and the logarithmic model is suitable for the situation with high density, otherwise there will be a large error. Usually, these situations are inconsistent with the actual traffic conditions. Compared with the car following model, the continuous medium model based on fluid dynamics theory could better explain the collective behavior of vehicle flow from a macro perspective, which used a dynamic method to analyze the situation of traffic flow. However, the application scope of the model based on fluid dynamics was limited. It seemed to be more suitable for dense, uniform, and steady traffic flow, but it could not do anything for free flow and intermittent flow. Even for the case of non-free flow, the model based on fluid dynamics cannot fully explain the phenomena of traffic congestion, going and stopping traffic flow, traffic jam, and vehicle flow instability [2]. These models encounter difficulties in explaining the data gap when the traffic flow approaches the capacity and the jumping change of traffic state. In view of the shortcomings of traditional modeling methods based on two-dimensional plane and continuous traffic flow relationship model, this paper will try to analyze this discontinuous jumping phenomenon of traffic flow state based on three-dimensional space.

When the traffic flow approached the capacity, there was a data gap, and there was a phenomenon that the traffic flow changed directly from the unblocked state to the congested state, or jumped from the congested state to the unblocked state. In the process of change, the traffic flow state show the characteristics of jumping state, multimodality, and bifurcation, which were consistent with the basic characteristics of the object studied by catastrophe theory. The catastrophe theory could reflect the change of traffic state from threedimensional space, and could more accurately and reasonably explain the change process of traffic state, which overcame the shortcomings of traditional continuous traffic flow model based on two-dimensional plane.

Some scholars applied the idea of catastrophe theory to the theoretical analysis and practical application of traffic flow, and have achieved some research results [4-8]. Dendrinos et al. used catastrophe theory to analyze the relationship between speed and flow [9]. Navin et al. used catastrophe theory to analyze the catastrophe process in traffic flow, and established cusp catastrophe model with traffic flow and occupancy as control variables. The model could well explain the observed data gap and traffic state jump phenomenon [10]. Hall et al. verified the traffic catastrophe model based on catastrophe theory with real traffic flow data, and gave the coordinate transformation from traffic flow data to catastrophe surface [11]. In China, He $\mathrm{X}$. first used the cusp catastrophe theory to establish a traffic flow model [12]. Zhang et al. used the data of the Pearl River Delta Expressway to verify the cusp catastrophe theory, analyzed the characteristics of traffic flow in the process of mutation, and used the catastrophe theory to predict the traffic flow [13]. Shui et al. analyzed the relationship between traffic bottleneck and traffic congestion on expressways, and proved that the traffic operation state would change suddenly in a short time and lead to traffic congestion [14].

Through the detection of freeway accidents and the classification of traffic conditions, Aultmanhall et al. established a freeway traffic operation model based on catastrophe theory to detect freeway traffic accidents [15]. Luo et al. established a three-dimensional traffic flow model to describe the change characteristics of the three parameters of traffic flow, verified that the three basic parameters of traffic flow had similar characteristics and change rules with cusp catastrophe theory, and further proved the feasibility of using cusp catastrophe theory to describe the relationship characteristics of the three basic parameters of traffic flow [16]. Carrillo et al. proved the mutation and bifurcation of traffic wave in the second order traffic flow model [17]. Aiming at the frequent congestion phenomenon of urban expressway, $\mathrm{Xu} \mathrm{L}$. et al. analyzed the evolution process of traffic congestion through the cusp catastrophe model [18]. Theofilatos used cusp catastrophe method to explore the frequency of traffic accidents on urban expressways based on real-time traffic and weather data [19]. Based on cusp catastrophe theory, Long et al. established a traffic flow representation model of urban road, and used outlier mining technology to detect traffic accidents [20]. This method can effectively distinguish conventional congestion and traffic accidents. Wang et al. analyzed the application prospect of catastrophe theory in the field of transportation [21]. Hu et al. divided the state of expressway traffic flow into two states: free flow and non-free flow, and thought that the transition between the two states was not a gentle gradual process. There was a short mutation in a certain speed range, which presented a chaotic state. Based on catastrophe theory, the traffic flow model of speed, flow, and time occupancy was established in three-dimensional space system [22].

In this paper, data gap and traffic state jump phenomenon were observed through traffic investigation. On the basis of analyzing the characteristics of traffic flow mutation, the cusp mutation model of traffic flow state was established to analyze the relationship between traffic flow parameters when traffic flow state mutation occurs. This paper discussed the relationship between different road grades, actual flow, road congestion density, speed, critical wave speed and traffic state, and verified it by an example.

Our primary contributions were summarized as follows: (1) we confirmed that the change of traffic state showed jumping. It was found that with the change of traffic flow, the whole road presented a process from smooth to congestion and then to smooth. In this process, it shows multiple equilibrium positions, and the external characteristics of traffic flow were consistent with the characteristics of 
catastrophe theory. (2) Based on the traffic wave model established by the basic principle of fluid dynamics, the catastrophe model of traffic flow was established, the stability of traffic flow system was analyzed, and the solution method of the model was given. (3) This paper analyzed the stability of traffic flow system, and expounded the method to judge the stability of the model. Through the cusp catastrophe model, the bifurcation set of structural stability of the model was determined, and the relationship between traffic flow parameters was analyzed when the state of traffic flow changes suddenly in different operating environments.

\section{Background}

2.1. Catastrophe Model. In the process of development and change, things often jump from one state to another, that is, there is a sudden change. The traditional gradualism thought cannot use calculus to establish this state change model of things and, therefore, there are some obstacles in explaining this kind of jump phenomenon. Catastrophe theory uses the basic principle of structural stability theory, uses mathematical model to describe the process of qualitative change caused by catastrophic interruption of continuous change, and analyzes the relationship between control factors when state mutation occurs [23-26]. It can well explain the catastrophe phenomenon in the development process of things, and is an effective method to study discontinuous and nonlinear problems [27-29].

Rene Thom, a French mathematician, first put forward the basic idea of catastrophe theory in the 1970s [13]. The theory uses the potential function to express the transition between stable state and unstable state, and uses the existence or disappearance of the potential function as the basis to judge the stability of the system [30-33]. When a depression exists in the potential function, it means that the system development process is in a stable state. When the depression disappears, it means that the system is in an unstable state. In the process of state transition, the equilibrium surface (also called catastrophe manifold) is used to describe the balance surface. By classification of equilibrium surfaces, seven kinds of elementary catastrophe mathematical models can be obtained, which are folding type, cusp type, swallow tail type, butterfly type, hyperbolic type, elliptic type, and parabolic type [26]. These models use potential function to express the relationship between state variables and control variables in catastrophe system. The most widely used one is cusp catastrophe model based on three-dimensional space [26], and its potential function is

$$
U(x, u, v)=x^{4}+u x^{2}+v x .
$$

The parameters meaning in (1) is shown in Table 1.

By deriving the potential function, the catastrophe equation, also known as the equilibrium surface equation, is

$$
\frac{\partial U}{\partial x}=4 x^{3}+2 u x+v=0 \text {. }
$$

TABle 1: Meaning of parameters in (1).

\begin{tabular}{lc}
\hline Parameter & Parameter meaning \\
\hline$U($ ) & Potential function \\
$X$ & System internal state variable \\
$U$ & External control variable \\
$V$ & Another external control variable \\
\hline
\end{tabular}

2.2. Catastrophic Characteristics. Catastrophe theory uses mathematical tools to analyze and discuss the phenomenon of jump changes in the local and global characteristics of the system state in the differential dynamic system [30-32]. For a sudden change system, the following characteristics are usually shown in the evolution process $[12,21,26]$ :

(i) Multimodality: there are many stable states in the evolution process of multimode system. The potential of the system may have two or more minima when the control variables change in different ranges. The system shows two or more states, which is multimodality.

(ii) Mutagenicity: when the control variables change, the system jumps from one stable state to another.

(iii) Inaccessible: there are some unstable states in the system. When the control parameters and external factors change, the system directly transits from a stable equilibrium state to another equilibrium state. In this process, there is at least one unstable equilibrium position, which is actually unreachable.

(iv) Divergence: when the control parameters of the system change slightly near the equilibrium position, the state variables of the system increase slightly, and the state change process of the system is gradual. When the control parameters change slightly in the neighborhood of the degradation critical point, the state variables will change greatly. And when the control parameters change in the unstable region, the small change of the control variables in different directions will cause completely different changes of the system state, which is called divergence or bifurcation.

(v) Hysteresis: when the control parameters of the system change in different directions, the position where the system state transitions is different. It is that the change process is irreversible, and there is a hysteresis phenomenon.

For the nonlinear catastrophe system and catastrophe phenomenon, there are several equilibrium stable states in the evolution process. The structure of cusp catastrophe model is shown in Figure 1.

Figure 1(a) shows the diagram of cusp catastrophe equilibrium surface, which was divided into upper lobe, middle lobe, and lower lobe. The upper lobe and lower lobe represented the stable region. In the middle lobe, the state of the system changed abruptly, which was the unstable region.

When the control parameters and external factors change, the system often jumps from one state to another. In 


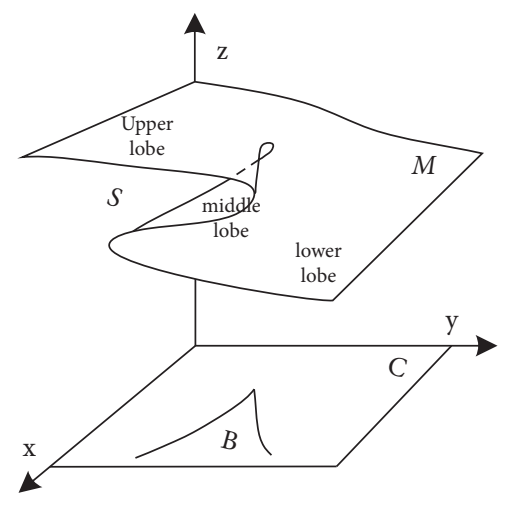

(a)

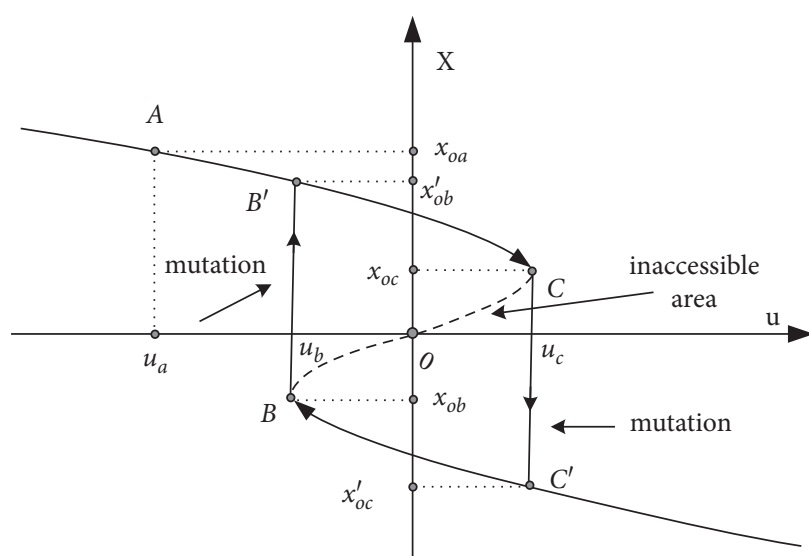

(b)

Figure 1: Diagram of cusp catastrophe model. (a) Cusp catastrophe equilibrium surface. (b) Schematic diagram of cusp mutation streamline.

this process, there are unreachable and unstable states. When the control parameters change slightly near the equilibrium position, the system state will appear the phenomenon of delay and bifurcation.

Figure 1(b) shows the epidemic structure of cusp mutation model. When point $\mathrm{A}$ on the curve moved to point $\mathrm{C}$, the state of the system changed continuously. When it reached point $\mathrm{C}$, as long as there was a small disturbance, the state of the system would change abruptly, and the state would jump from point $C$ to point $C^{\prime}$. In the process of moving from point $C^{\prime}$ to point $B$, the state of the system was in the lower half of the surface, and the system is stable. When it reached point $B$, if there was a small disturbance, the state of the system would undergo a sudden change, jumping to the $\mathrm{B}^{\prime}$, which was in the upper lobe. In fact, the state of the system cannot reach this region. On the surface $M$, when the control variables change along different paths, the state values of the system will be different and have multimodality. When the control variables change in different directions near the critical range, the position of system state mutation was different and has hysteresis. When the control parameters were located in the upper lobe, the small disturbance near $u_{c}$ made the system state change abruptly at point $\mathrm{C}$. When the control parameter was in the lower lobe, the small disturbance near $u_{b}$ made the traffic flow state change suddenly at point $B$.

Through the critical equilibrium surface equation, the stability of the extreme value of the system potential function can be analyzed, and the change trend of the extreme value of the system can be determined.

\section{Analysis of Sudden Change Characteristics of Traffic Flow State}

The transportation system is a typical nonlinear system, which has great randomness. The research group collected the traffic data of an expressway in Guangzhou and drew the scatter plot of flow and density, as shown in Figure 2. We divided the traffic flow into four states, which are free flow

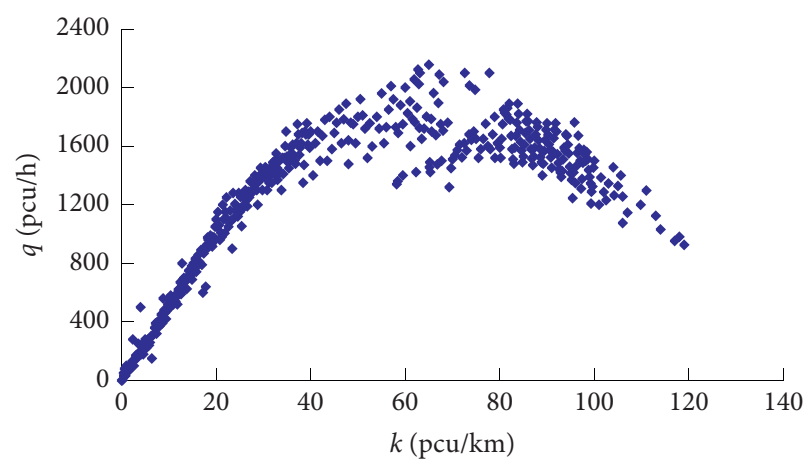

FIgURE 2: Flow-density scatter plot.

(state 1), steady flow (state 2), congested flow (state 3 ), and severe congestion state (state 4), as shown in Figure 3. With the change of traffic flow, the whole road presents a process from smooth to congestion and then to smooth, showing multiple equilibrium positions, which reflects the polymorphism of the system in catastrophe theory. With the increase of traffic flow, the density of traffic flow increases gradually, the speed decreases continuously, and the whole road is still in a stable state. It is a process of gradual change. When the traffic flow is close to the capacity of the road, if there is minor interference, such as a traffic accident, the speed of the vehicle will suddenly drop, the density will increase sharply in a short time, and the road will change from a smooth state to a crowded state, and even lead to congestion.

Through a large number of traffic survey data, we patched and processed the missing data, and got the flow density curve. It can also be seen from the curve that there was a data gap, in which there were very few or no data points [21]. This phenomenon shows that the traffic state changed directly from the stable and smooth state to the congested state. In this process, the traffic state had the characteristics of mutation. Capacity was the maximum flow of traffic on the road when the road was in good condition, which was an ideal condition. When the traffic flow is near 


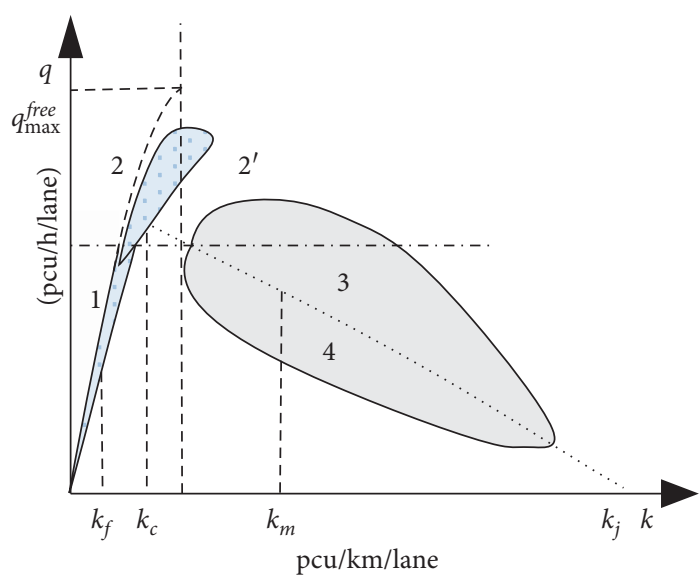

Figure 3: State division of traffic flow.

the capacity, the system was extremely unstable and the data points collected were few. It shows that the system was not reachable. At this time, the state of traffic flow was in a critical equilibrium state [34]. If the road running condition changes to the favorable condition, the speed of traffic flow would increase slowly, and the road would keep smooth. If there was traffic interference, the road would change from an unblocked state to a congested or blocked state. The speed would drop sharply, and the traffic condition would deteriorate rapidly. This phenomenon was consistent with the divergence (bifurcation) of catastrophe theory. These characteristics indicated that the traffic flow state was consistent with the characteristics of the catastrophe theory, and catastrophe theory could be used to analyze the discontinuity of the traffic flow.

\section{Traffic Flow Catastrophe Model Based on Cusp Catastrophe}

4.1. Model Establishment. The occurrence of traffic congestion is the result of the intensification of the contradiction between road capacity and actual traffic flow demand on the road. The capacity of the road is not fixed, but is affected by the road structure, traffic environment and traffic incidents. The width of the road or the number of lanes decreases, and the traffic capacity decreases. As shown in Figure 4, in the ao section, the number of lanes is 4 , and in the $o b$ section, the number of lanes is 3 . The capacity changes sharply at $o$ point, which leads to the change of normal operation state.

Assume that there is a vertical line $\mathrm{S}$ at point $o$, which divides the upstream and downstream into two regions with different densities, $\mathrm{A}$ and $\mathrm{B}$. The density of traffic flow in region $\mathrm{A}$ is $k_{1}$ and the speed is $v_{1}$, while the density of traffic flow in region $\mathrm{B}$ is $k_{2}$, and the speed is $v_{2}$. According to the basic theory of traffic waves, when near point $o$, the traffic flow will be chaotic. Due to the sudden drop of traffic capacity, the speed of vehicles in region A decreases, and traffic congestion will begin to develop. This state gradually spreads upstream at a certain speed and propagates backward like the wave of fluid. In this process, $S$ acts as the interface,

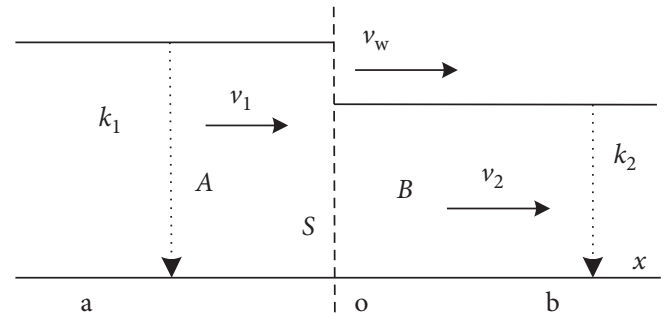

FIgURE 4: Road structure diagram.

which is called the wave front, and the moving speed at $S$ is $v_{w}$.

According to the relationship between the three parameters of traffic flow:

$$
\begin{aligned}
& q_{1}=v_{1} k_{1}, \\
& q_{2}=v_{2} k_{2} .
\end{aligned}
$$

According to the conservation of traffic flow, the number $N$ of vehicles entering and leaving the boundary line $S$ in time $t$ is equal. It is

$$
\begin{aligned}
N & =\left(v_{1}-v_{w}\right) k_{1} t_{1} \\
& =\left(v_{2}-v_{w}\right) k_{2} t .
\end{aligned}
$$

The meaning of the parameters in (3) is shown in Table 2.

According to (3) and (4), the propagation velocity of wave front $\mathrm{S}$ is obtained as follows:

$$
v_{w}=\frac{q_{2}-q_{1}}{k_{2}-k_{1}} .
$$

In the case that the traffic volume and density of the regions $\mathrm{A}$ and $\mathrm{B}$ do not change very much, $v_{w}$ can be expressed as follows:

$$
v_{w}=\frac{\triangle q}{\triangle k}=\frac{d_{q}}{d_{k}}=\frac{q}{k}+k \frac{d_{v}}{d_{k}}=v+k \frac{d_{v}}{d_{k}} .
$$

According to the generalized velocity-density model, the relationship between velocity and density can be expressed as follows:

$$
v=v_{f}\left[1-\left(\frac{k}{k_{j}}\right)^{s+1}\right] .
$$

In (7), $v_{f}$ is the free flow velocity, $k_{j}$ is the blocking density, $s$ is the parameter, and $s>0$. The following equation can be obtained by solving the differential of (7),

$$
\frac{\mathrm{d} v}{\mathrm{~d} k}=-\frac{(s+1) v_{f} s}{k_{j}^{s+1}} k^{s} .
$$

When $s=1$, by substituting (8) into (6), we can get

$$
k^{3}+\frac{k_{j}^{2}}{2 v_{f}} v_{w} k-\frac{k_{j}^{2}}{2 v_{f}} q=0 .
$$

This equation is similar to the equilibrium surface (2) of cusp catastrophe model. This equation is taken as the critical 
TABLE 2: Meaning of parameters in equations (3) and (4).

\begin{tabular}{lcc}
\hline Parameter & Parameter meaning & $\mathrm{Unit}$ \\
\hline$N$ & Standard vehicle number & $\mathrm{pcu}$ \\
$q_{\mathrm{i}}(i=1,2)$ & Traffic volume & $\mathrm{pcu} / \mathrm{h}$ \\
$v_{i}(i=1,2)$ & Traffic flow velocity & $\mathrm{m} / \mathrm{s}$ \\
$k_{\mathrm{i}}(i=1,2)$ & Traffic flow density & $\mathrm{pcu} / \mathrm{km}$ \\
$v_{w}$ & Traffic wave velocity & $\mathrm{m} / \mathrm{s}$ \\
$T$ & Running time & $\mathrm{s}$ \\
\hline
\end{tabular}

equilibrium surface, the traffic flow density $k$ is taken as the state variable, and the traffic wave propagation speed $v_{w}$ and the traffic flow $q$ are considered as the control variables. The cusp catastrophe model of traffic flow can be constructed as follows:

potential function : $E\left(k, v_{w}, q\right)=\frac{1}{4} k^{4}+\frac{k_{j}^{2}}{2 v_{f}} v_{w} k^{2}-\frac{k_{j}^{2}}{2 v_{f}} q k$,

equilibrium surface $\mathrm{M}: \frac{\partial E}{\partial k}=k^{3}+\frac{k_{j}^{2}}{2 v_{f}} v_{w} k-\frac{k_{j}^{2}}{2 v_{f}} q=0$.

4.2. System Stability Analysis. The equilibrium surface is a univariate cubic equation with density $k$ as the unknown variable. When the propagation speed of traffic wave $v_{w}$ and traffic flow $q$ change, the state variable $k$ changes continuously. The curve change trend of state variable in $R^{2}$ space can be obtained from (11), as shown in Figure 5.

It can be seen from Figure 5 that when $v_{w}>0$, the critical equilibrium curve is an anti-S curve with gentle change, that is curves (a) and (b) in Figure 5. Because of $v_{w}>0$, the propagation direction of traffic wave is consistent with the running direction of traffic flow. The traffic flow increases gradually, but the density of traffic flow decreases gradually. At this time, the traffic wave is a diverging wave, and the propagation direction of the wave is consistent with the direction of the traffic flow, which is a diverging wave. When $v_{w}=0$, the critical equilibrium curve (c) is shown in Figure 5, which is an anti-s curve flow with small fluctuation. At this time, the traffic state is in a critical equilibrium state. The flow is large and close to the capacity of the road. If there is interference from the external environment, the traffic flow state will change suddenly, the speed will drop sharply, and the density will increase, which will lead to the occurrence of traffic congestion. If the traffic disturbance is eliminated in time, the congested traffic flow will be gradually dispersed, and the traffic state will change from unstable to stable operation state, but at this time, the traffic flow density is high and the speed is low. When $v_{w}<0$, the critical equilibrium curves are (d) and (e) in Figure 5. With the change of traffic flow, the traffic

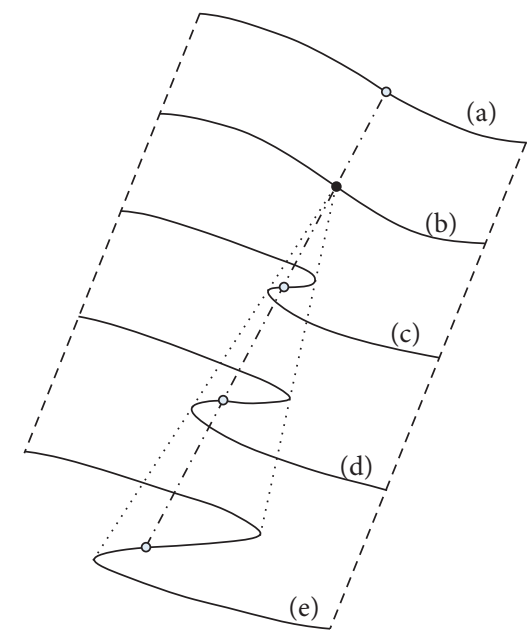

Figure 5: Cross-sectional curve diagram of critical equilibrium surface.

flow density has multimode and is jumping. Because of $v_{w}$ $<0$, the traffic wave is an aggregation wave, which indicates that the congestion gradually appears on the road and propagates to the upstream. The curve has great volatility, and the road traffic flow state is extremely unstable.

\section{Model Solution and Stability Judgment}

5.1. Bifurcation Set of Model Structure Stability. According to (11), when the control variables $v_{w}$ and $q$ were constantly changing, the change trend of the state variable $k$ could be observed, and the catastrophe flow pattern of the traffic flow cusp catastrophe model could be drawn. We got the critical equilibrium surface of three-dimensional space, as shown in Figure 6 .

It can be seen from Figure 6 that the equilibrium surface $M$ was a curved surface with folds, which can be divided into three parts: upper lobe, middle lobe, and lower lobe. On the surface $M$, when $(\partial E / \partial k)=0$ and $\left(\partial^{2} E / \partial k^{2}\right)>0$, the state points were located in the region of upper and lower lobes. When $(\partial E / \partial k)=0$ and $\left(\partial^{2} E / \partial k^{2}\right)<0$, the state points were located in the region of middle lobe. On the equilibrium surface, the upper and lower lobes represented the stable equilibrium state of the system, and the middle lobes represented the unstable state. When the control variable changed, the system state variable $k$ would change, and the trajectory of the curve would also change. When $v_{w}$ and $q$ changed in the lower and upper lobes, there was a unique state value $k$ in the system, and the change process of the system is a gradual and stable process. When the trajectory passed through the folding area, the system was in an unstable state, and the state mutation was easy to occur at the folding part of the fold. The singular point set $\mathbf{S}$ was obtained by quadratic differentiation of the potential function, where the state of the system changed abruptly. The set $\mathbf{S}$ can be expressed as follows: 


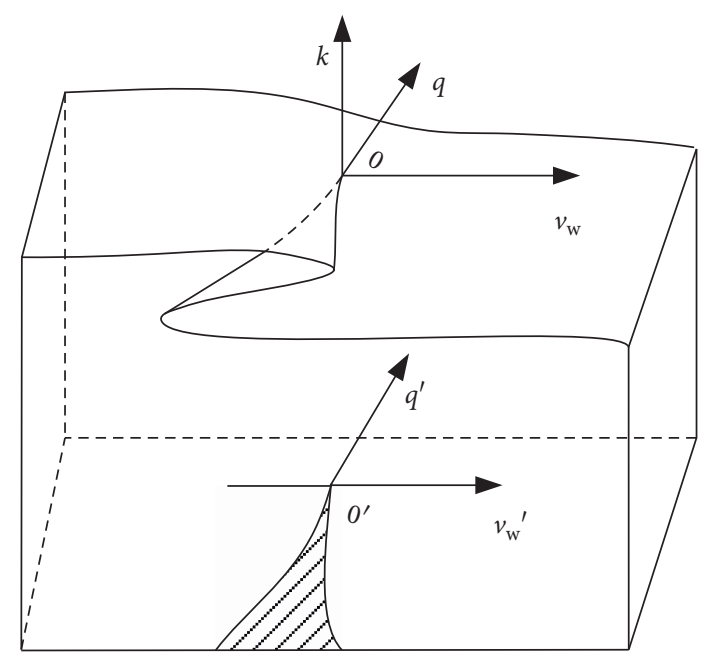

Figure 6: Traffic density cusp catastrophic model equilibrium surface.

$$
\left\{\begin{array}{l}
\frac{\partial E}{\partial k}=k^{3}+\frac{k_{j}^{2}}{2 v_{f}} v_{w} k-\frac{k_{j}^{2}}{2 v_{f}} q=0 \\
\frac{\partial^{2} E}{\partial k^{2}}=3 k^{2}+\frac{k_{j}^{2}}{2 v_{f}} v_{w}=0 .
\end{array}\right.
$$

By eliminating the state variable $k$ in (12), the bifurcation set $B$ of the stability of the traffic flow state structure can be obtained:

$$
\frac{1}{2}\left(\frac{k_{j}^{2} v_{w}}{v_{f}}\right)^{3}+\frac{27}{4}\left(\frac{k_{j}^{2} q}{v_{f}}\right)^{2}=0
$$

According to (12), the singularity set $S$ was projected on the control plane, and the graph of the bifurcation set $\boldsymbol{B}$ can be obtained, as shown in Figure 7.

As can be seen from Figure 7, the bifurcation set $\boldsymbol{B}$ is a symmetrical parabolic curve $L_{1}$ and $L_{2}$, and the shadow areas $A_{2}$ and $A_{3}$ correspond to the folding area (i.e. the middle lobe) of the equilibrium surface. In practice, due to the traffic flow $q \geq 0$, the area suitable for the traffic flow model is the positive half axis part of the folding area, and the corresponding area is $A_{3}$.

5.2. Model Solution and System Stability Judgment. The equation of equilibrium surface (11) is a cubic equation in one unknown, and its real root of the solution was one or three, which was determined according to the discriminant $\Delta$.

Define

$$
\Delta=\left(\frac{k_{j}^{2} q}{4 v_{f}}\right)^{2}+\left(\frac{k_{j}^{2} v_{w}}{6 v_{f}}\right)^{3} .
$$

Based on (14), the number of solutions of the system can be judged by the value of the discriminant $\Delta$. The

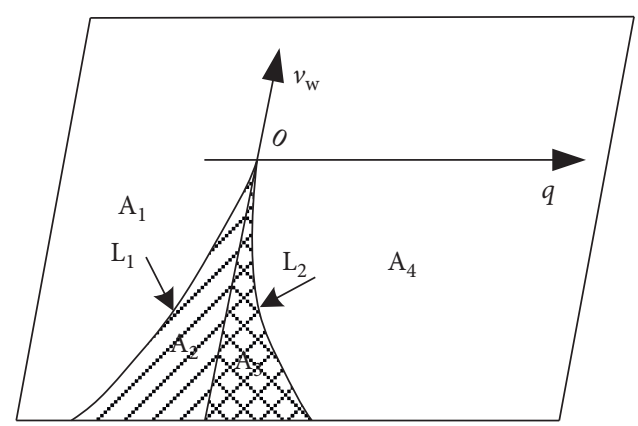

FIgURE 7: Bifurcation set diagram of mutations surface.

relationship between the solution of the system, bifurcation set $\boldsymbol{B}$ in Figure 7 and system stability is shown in Table 3.

When $\Delta>0$, the system had only one solution, which was in the stable area of the system. When the control variable changed in this area, the traffic flow state was stable. If the control variable $v_{w}>0$, with the change of $v_{w}$ and $q$, the change process of the state variable $k$ was gradual, and the system was in a noncongested state. At this time, the traffic wave was divergent wave, and the propagation direction of the wave was consistent with the running direction of the traffic flow. If the control variable $v_{w}<0$ and $\Delta>0$, the density $k$ was gradual. The traffic wave was an aggregation wave, and the propagation direction of traffic wave was opposite to that of traffic flow. At this time, the vehicle speed decreased, and the density increased gradually, but the road traffic was still in a smooth state.

When $\Delta=0$, it was the critical stability curve of the system state. When the control variable had a small disturbance on or near the curve, the traffic flow state would change abruptly. At this time, the road traffic state was at the edge of smooth and crowded. The slight change of traffic running environment would lead to the qualitative change of traffic flow state. If the traffic environment at that time was conducive to the running of vehicles, the road would be in a smooth state. If the traffic environment at that time developed in the direction that was not conducive to the running of vehicles, the traffic density would continue to increase, and the speed would drop rapidly. In this way, the traffic situation on the road would continue to deteriorate, and the traffic state would turn into a congested state.

When $\Delta<0$, the system had three solutions, the state of the system was uncertain, which corresponded to the unstable region of the system. When the change of control variable made the discriminant from $\Delta=0$ to $\Delta<0$, the density $k$ would change abruptly, and the value would change greatly. The traffic state trajectory point would jump from the lower lobe of the equilibrium surface to the upper lobe. At this time, the slight change of traffic flow or traffic wave speed wave speed on the road would make the density of traffic flow jump, and the density would suddenly increase, which would lead to the rapid decline of traffic flow speed, and the road would be congested or even blocked.

This phenomenon was consistent with the actual running of road vehicles, and the existence of data gap of traffic flow parameters by the actual observation was confirmed, 
TABLE 3: Relationship between system solution and system state.

\begin{tabular}{lccc}
\hline$\Delta$ value & Number of system solutions & Correspondence with bifurcation set $B$ in Figure 7. & Status of the system \\
\hline$\Delta>$ & 1 & Area A1, A4 & Stable \\
$\Delta=0$ & - & Curves L1, L2 & Critical stable \\
$\Delta<0$ & 3 & Area A2, A3 & Unstable \\
\hline
\end{tabular}

and the reason of traffic flow mutation could be explained theoretically.

\section{Traffic Flow State Mutation Analysis}

When the road traffic running environment was different, the critical range of traffic flow state mutation was also different. From $\Delta=0$, we could get the following:

$$
\begin{gathered}
v_{w c}=-\sqrt[3]{\frac{27 v_{f} q^{2}}{2 k_{j}^{2}}}, \\
k_{c}=\sqrt[3]{\frac{2 k_{j}^{2} q}{v_{f}}} .
\end{gathered}
$$

The meaning of the parameters in (15) is shown in Table 4

It can be seen from (15) that when $\mathbf{v}_{\mathbf{f}}, \boldsymbol{k}_{\boldsymbol{j}}$, and $\boldsymbol{q}$ were different (i.e. different traffic environments), the critical range of abrupt change of traffic flow state was also different. For different grades of roads, the design speed was different, and the free flow speed $\mathbf{v}_{\mathbf{f}}$ was different when the flow was small. For roads with different service levels, the congestion density $\boldsymbol{k}_{\boldsymbol{j}}$ was different under congestion conditions.

The critical range of traffic parameters and their relationship under different running environments would be analyzed and discussed in three cases, as shown in Table 5.

\subsection{The Relationship between Critical Wave Velocity and Flow} under Different Road Grades. If the grade of the road was high, the design speed was also large, and the free flow speed of the vehicle driving on the road is also large. When the road blocking density $k_{j}=125 \mathrm{pcu} / \mathrm{km}$, the relationship between the critical wave velocity and flow at different road levels is shown in Figure 8. Vw1-Vw4 are the critical velocities when $v_{f}$ were $50,60,80$, and $100 \mathrm{~km} / \mathrm{h}$ respectively.

In Figure 8, it could be seen that under the same road grade, the traffic wave $v_{w}$ increased with the increase of traffic flow, that is to say, the faster the propagation speed of the affected traffic flow was, it was easy to cause traffic congestion. When the road grade was different, the critical wave velocity $v_{w c}$ was different under the condition of the same flow $q$. The higher the road grade was, the greater the design speed was, and the greater the traffic wave $v_{w}$ was. In the case of traffic interference, the higher the vehicle speed was, the faster the deceleration of the front of the vehicle affected the speed change of the vehicles behind, which led to a sharp drop in the speed of the traffic flow on the road in a short time. The traffic volume would decrease rapidly and
TABLE 4: Meaning of parameters in (15).

\begin{tabular}{lcc}
\hline Parameter & Parameter meaning & Unit \\
\hline$Q$ & Traffic volume & $\mathrm{pcu} / \mathrm{h}$ \\
$v_{f}$ & Free flow velocity & $\mathrm{m} / \mathrm{s}$ \\
$v_{w c}$ & Critical traffic wave velocity & $\mathrm{m} / \mathrm{s}$ \\
$k_{\mathrm{j}}$ & Blocking density & $\mathrm{pcu} / \mathrm{km}$ \\
$k_{\mathrm{c}}$ & Critical density & $\mathrm{pcu} / \mathrm{km}$ \\
\hline
\end{tabular}

the traffic density would increase gradually. The possibility of traffic congestion caused by sudden change of traffic flow state was very large.

6.2. The Relationship between the Critical Wave Speed and the Traffic Flow at the Same Speed. When the free flow velocity $V_{\mathrm{f}}$ was $80 \mathrm{~km} / \mathrm{h}$ and the blocking density $k_{j}$ was $100,125,150$, and $200 \mathrm{pcu} / \mathrm{km}$, respectively, the relationship curve between critical wave velocity and flow is shown in Figure 9.

It could be seen from Figure 9 that if the free flow velocity $v_{f}$ was 80 , the critical wave velocity $v_{w 1}$ when $k_{j 1}$ was 100 was greater than $v_{w 4}$ when $k_{j 4}$ was 125 . But when traffic interference was encountered, the actual $v_{w 1}$ is less than $v_{w 4}$, which indicated that the smaller the blocking density $k_{j}$ was, the smaller is the possibility of traffic mutation. This kind of situation often occurred in the real road traffic running. When there was traffic disturbance, the speed of the traffic flow decreased and spread to the upstream at a certain speed, which gradually affected the speed of the vehicles behind. In the case of disturbance elimination, the road system would gradually return to normal according to its own adjustment ability. If the traffic disturbance could not be eliminated by itself, corresponding traffic control measures should be taken. Otherwise, the traffic state will continue to deteriorate, the flow would be further reduced, and the vehicle density on the road would gradually increase. When it reached the critical value, the state mutation would occur, which would lead to the occurrence of traffic congestion.

6.3. Relationship between Critical Density and Flow under Different Service Levels. When $k_{j}$ was $125 \mathrm{pcu} / \mathrm{km}, k_{c 1} \sim k_{c 4}$ were the variation curves of traffic flow and critical density when the free flow speed $v_{f}$ was $50,60,80$, and $100 \mathrm{~km} / \mathrm{h}$, respectively, as shown in Figure 10.

It can be seen from Figures 8 to 10 that in the case of large flow, the probability of traffic state mutation of roads with higher road grade was higher than that of roads with lower road grade. The reason was that the higher the road grade, the faster the running speed. When there was a small disturbance, it had a great influence on the velocity. At this time, the propagation speed of traffic wave was very fast, and 
TABLE 5: Case comparison of different running environments.

\begin{tabular}{lcc}
\hline Case & Running environment description & $\begin{array}{c}\text { Analyze the relationship between } \\
\text { parameters }\end{array}$ \\
\hline A & Same blocking density $k_{j}=125 \mathrm{pcu} / \mathrm{km}$, different free flow velocity $v_{f}=50,60,80,100 \mathrm{~km} / \mathrm{h}$ & $V_{w c}$ and $q$ \\
B & Same free flow velocity $v_{f}=80$, different blocking density $k_{j}=100,125,150,200 \mathrm{pcu} / \mathrm{km}$ & $V_{w c}$ and $q$ \\
C & Same blocking density $k_{j}=125 \mathrm{pcu} / \mathrm{km}$, different free flow velocity $v_{f}=50,60,80,100 \mathrm{~km} / \mathrm{h}$ & $k_{c}$ and $q$ \\
\hline
\end{tabular}

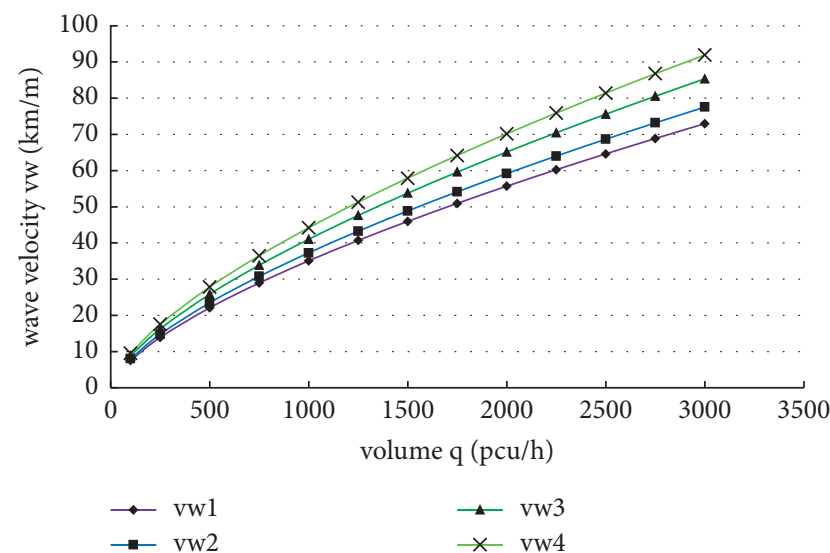

FIGURE 8: $\mathbf{k}_{\mathbf{j}}=125$, relationship between critical wave velocity $\mathbf{v}_{\mathbf{w c}}$ and flow $\mathbf{q}$ under the condition of different highway grades.

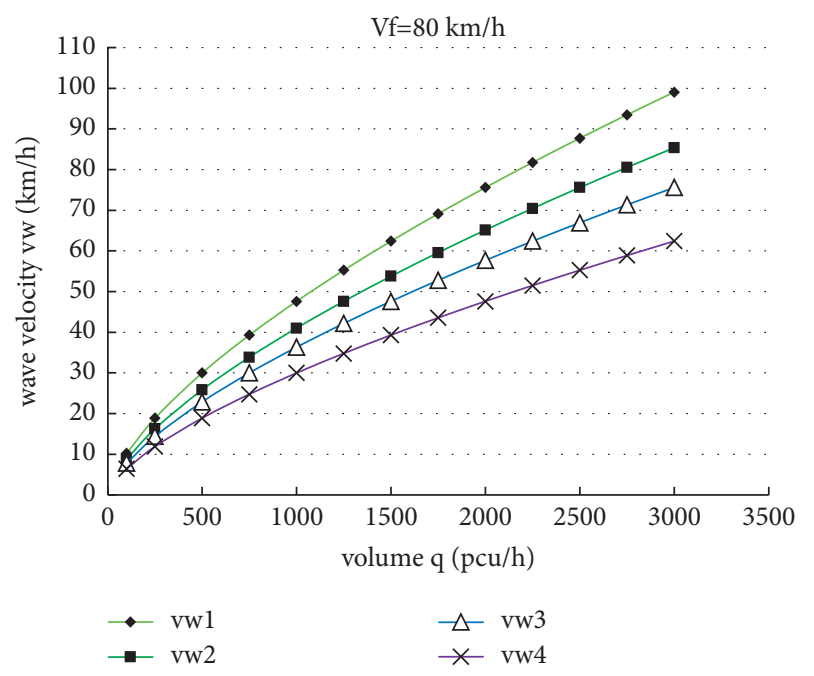

FIGURE 9: $\mathbf{v}_{\mathbf{f}}=80$, the relationship between critical velocity $\mathbf{v}_{\mathbf{w c}}$ with flow $\mathbf{q}$ under different block densities.

more and more vehicles were affected, which led to a significant decrease in the speed of traffic flow in a short time and a rapid increase in the density of vehicles on the road. This would lead to traffic congestion. For example, when $k_{j}$ is $125 \mathrm{pcu} / \mathrm{km}$, vw4 in Figure 8 was the traffic wave speed and the free flow speed $v_{f}$ was $100 \mathrm{~km} / \mathrm{h}$. The free flow speed was larger than others, and the traffic wave vw4 was also larger than vw1, vw2, and vwv3. While in Figure 8, the critical density $k_{c}$ was the smallest at this free flow speed, so the sudden change of traffic state was most likely to occur.

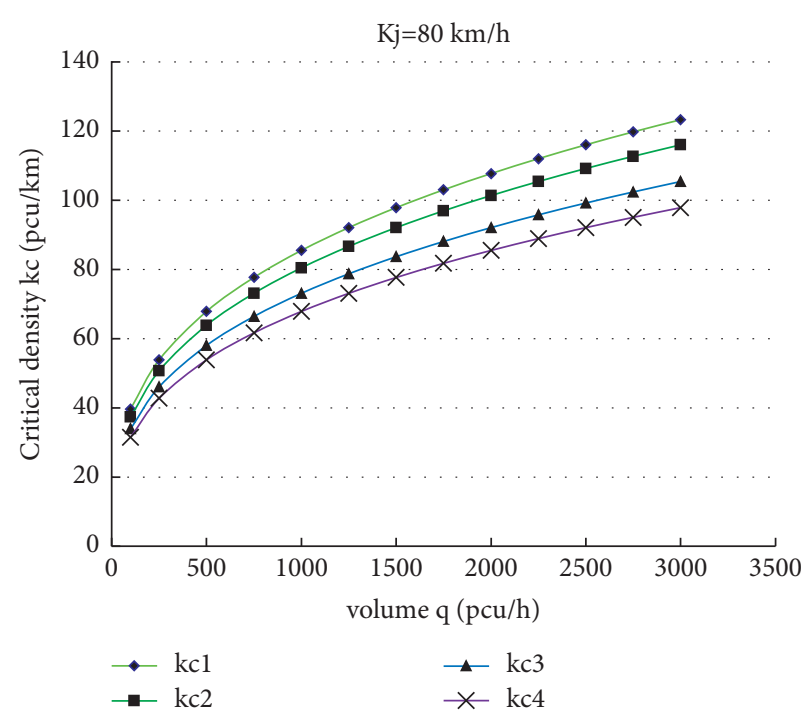

FIgURE 10: The critical density $k_{c}$ and flow $q$ relationship.

But in the lower grade road, the vehicle speed was low, the density was large, and the whole road running state was relatively stable. If there was traffic interference, because the vehicle speed was small, the speed drop was relatively small, the traffic propagation speed was slow, and the influence of the traffic flow on the whole road was small. At this time, the speed of the whole road traffic flow would be reduced, the flow would drop, the whole road could maintain a smoothrunning state, and the sudden change of traffic state was impossible. For example, in Figure 8, the curve vw1 was the traffic wave speed when the free flow speed $v_{f}$ was $50 \mathrm{~km} / \mathrm{h}$. The free flow speed was the smallest, and the traffic wave speed vw1 was also the smallest. In Figure 8, the critical density $k_{c}$ was the largest at this free flow speed, so the possibility of traffic state mutation was the smallest.

6.4. Case Analysis. We took Guangyuan Expressway in Guangzhou as an example to analyze the traffic flow state. Guangyuan expressway was a high standard urban expressway in Guangzhou. The total length was $47 \mathrm{~km}$, with two-way six lanes. The intersection was in the form of full interchange. The design speed was $60 \mathrm{~km} / \mathrm{h}$ on the ground and $80 \mathrm{~km} / \mathrm{h}$ on viaduct. The straight-line distance between each two toll stations was $2-3.5 \mathrm{~km}$. Detectors were set at the entrance and exit to detect traffic flow, occupancy, speed and other traffic parameters. We selected some sections of the expressway as the research object, sorted out the original 
TABLE 6: Road traffic flow status.

\begin{tabular}{|c|c|c|c|c|c|}
\hline Time & Speed $v(\mathrm{~km} / \mathrm{h})$ & Flow $q(\mathrm{pcu} / \mathrm{h})$ & Position in equilibrium surface & Traffic flow status & Road traffic state \\
\hline $6: 30$ & 56.52 & 503 & Upper lobe & Stable & Smooth flow \\
\hline $6: 45$ & 45.39 & 890 & Upper lobe & Stable & Smooth flow \\
\hline $7: 00$ & 41.68 & 1856 & Upper lobe & Stable & Stable flow \\
\hline $7: 15$ & 38.52 & 2030 & Fold & Critical stable & Slight congestion \\
\hline $7: 30$ & 28.74 & 2210 & Middle lobe & Unstable & Congested flow \\
\hline $7: 45$ & 20.65 & 2190 & Middle lobe & Unstable & Congested flow \\
\hline $8: 00$ & 34.82 & 1970 & Fold & Critical stable & Congested flow \\
\hline $8: 15$ & 39.97 & 2120 & Lower lobe & Stable & Stable flow \\
\hline $8: 45$ & 42.15 & 2010 & Lower lobe & Stable & Stable flow \\
\hline $9: 00$ & 54.26 & 1690 & Upper lobe & Stable & Smooth flow \\
\hline
\end{tabular}

data obtained from the entrance and exit of different sections, and removed the incomplete and wrong data [35].

We divided traffic flow into four states: free flow, steady flow, slight congested flow, and congested flow. From a large number of observation data, we selected the traffic flow data when different states changed, such as smooth flow to stable flow, slightly congested flow to congested flow, congested flow to blocked flow, blocked flow to stable flow and so on. These data were sorted out, and the stability and change trend of traffic flow state were analyzed by using the established traffic flow cusp catastrophe model.

Through the real-time observed traffic flow data, the critical stability region of the system was analyzed by simulation calculation, and the road traffic flow state was judged. The results are shown in Table 6 .

\section{Conclusion}

Through the observation and analysis of traffic flow detection data, we found the existence of traffic flow data gap and the state jump phenomenon when the traffic state changed, which show the basic characteristics of cusp catastrophe model. Based on the movement process of traffic flow, the cusp catastrophe model of traffic flow was established, and the stability of traffic flow state was analyzed. The sudden change characteristics of traffic flow state in different running environments were analyzed, and the change relationship between traffic flow parameters was discussed. Through case analysis, the traffic state analyzed by the cusp catastrophe model was consistent with the phenomenon of traffic flow under the actual traffic running environments. The results show that the cusp catastrophe model could describe the relationship between the three parameters of traffic flow with threedimensional space and could effectively analyze the internal relationship of parameters when the traffic flow state changed, which made up for the shortcomings of traditional two-dimensional plane analysis. The traffic flow cusp catastrophe model could effectively explain the traffic state jump phenomenon.

Our next research work is to further explore the application of catastrophe theory in the field of transportation. First, the evolution process of sudden change of road traffic flow state under different traffic running environments will be analyzed and discussed. Then, the catastrophe model would be used to judge the overall traffic flow running status on the road, the changing trend of traffic flow parameters under road unblocked state, stable state, and congested state will be analyzed, and the future traffic running state will be predicted.

\section{Data Availability}

The data used to support this study are available from the corresponding author upon request.

\section{Conflicts of Interest}

The authors declare that they have no conflicts of interest.

\section{Acknowledgments}

This work was supported in part by the National Natural Science Foundation of China (72061016), China National Scholarship Committee (201908360225), and Jiangxi Provincial Department of Education (GJJ160608).

\section{References}

[1] H. Hong and W. Guang, "Recent developments of traffic flow," Theory Journal of Transportation Systems Engineering and Information Technology, vol. 6, no. 1, pp. 80-85, 2006.

[2] W. D. Feng, G. G. He, and B. Liu, "A review of traffic flow theory," Journal of Systems Engineering, vol. 13, no. 3, pp. 71-82, 1998.

[3] M. H. Lighthill, "Whitham, on Kinematics Wave: II. A theory of traffic on long crowded roads," Proc.R.Soc. London,Ser.A, vol. 22, pp. 317-345, 1955.

[4] S. Ahn, S. Vadlamani, and J. Laval, "A method to account for non-steady state conditions in measuring traffic hysteresis," Transportation Research Part C: Emerging Technologies, vol. 34, pp. 138-147, 2013.

[5] G. J. Forbes and F. L. Hall, "The applicability of catastrophe theory in modelling freeway traffic operations," Transportation Research Part A: General, vol. 24, no. 5, pp. 335-344, 1990.

[6] J. A. Acha-Daza and F. L. Hall, "Application of catastrophe theory to traffic flow variables," Transportation Research Part B: Methodological, vol. 28, no. 3, pp. 235-250, 1994.

[7] A. Papacharalampous and E. Vlahogianni, "Modeling microscopic freeway traffic using cusp catastrophe theory," IEEE Intelligent Transportation Systems Magazine, vol. 6, no. 3, pp. 6-16, 2014. 
[8] W. Zhan, Y. Q. Shang, and F. X. Chi, "Application of catastrophe theory in traffic flow of highway tunnel group," Advanced Materials Research, vol. 734-737, pp. 1609-1612, 2013.

[9] D. S. Dendrinos, "Operating speeds and volume to capacity ratios: the observed relationship and the fold catastrophe," Transportation Research, vol. 12, no. 3, pp. 191-194, 1978.

[10] F. P. D. Navin, "Traffic congestion catastrophes," Transportation Planning and Technology, vol. 11, no. 1, pp. 19-25, 1986.

[11] F. L. Hall, "An interpretation of speed-flow-concentration relationships using catastrophe theory," Transportation Research Part A: General, vol. 21, no. 3, pp. 191-201, 1987.

[12] X. He, "The catastrophic model in traffic flow," Transactions of Changsha Communication College, vol. 2, pp. 81-85, 1986.

[13] Y. P. Zhang and Y. L. Pei, "Research on traffic flow forecasting model based on cusp catastrophe theory," Journal of Harbin Institute of Technology, vol. 11, no. 1, pp. 1-5, 2004.

[14] Y. Shui, C. Wei, and H. Zhen, "Research of Highway Bottlenecks Based on Catastrophe Theory," in Proceedings of the 2015 International Conference on Transportation Information and Safety (ICTIS), pp. 138-142, Wuhan, China, June 2015.

[15] L. Aultman-Hall, L. Hall-Fred, Y Shi, and B. Lyall, "A catastrophe theory approach to freeway incident detection," Applications of Advanced Technologies in Transportation Engineering, ASCE, Reston, VA, US, 2015.

[16] Q. Luo, J. YUAN, Y. J. HUANG, Z. X. DENG, and Y. M. TANG, "Study on traffic flow parameters and case analysis based on cusp catastrophe theory," Journal of Guangxi University, vol. 43, no. 6, pp. 2341-2346, 2018.

[17] F. A. Carrillo, S. Patricia, M. V. Rosa, and V. Fernando, "Traveling waves, catastrophes and bifurcations in a generic second order traffic flow model," International Journal of Bifurcation \& Chaos, vol. 12, no. 23, pp. 1294-1296, 2013.

[18] L. Xu and X. Wang, "Research on traffic flow congestion space-time evolution of urban expressway based on cusp catastrophe theory," Highways, vol. 12, pp. 133-144, 2016.

[19] A. Theofilatos, "Utilizing real-time traffic and weather data to explore crash frequency on urban motorways: a cusp catastrophe approach," Transportation Research Procedia, vol. 41, pp. 471-479, 2019.

[20] Q. Long, L. Hu, J. Zhang, and Z. Zhou, "Traffic incident detection based on the cusp catastrophe theory model," China Civil Engineering Journal, vol. 9, pp. 112-116, 2015.

[21] Y. Wang, D. Wang, S. Yang, and B. Dong, "A comprehensive review over the application of catastrophe theory in traffic flow," Journal of Transportation Systems Engineering and Information Technology, vol. 5, no. 6, pp. 68-71, 2005.

[22] J. R. Hu and L. He, "Freeway traffic flow condition criterion method based on cup catastrophe theory," China Journal of Highway and Transport, vol. 30, no. 10, pp. 137-144, 2017.

[23] W. U. Chen and L. Cheng, "Application of catastrophe theory in urban passenger transport structure evaluation," Journal of Transportation Systems Engineering and Information Technology, vol. 10, no. 4, pp. 166-171, 2010.

[24] G. C. Ao, Y. H. Jia, L. Jiangye, and H. L. Zhang, "Model of mixed motor vehicles flow parameters based on cusp catastrophe theory," System Engineering Theory \& Practice, vol. 29, no. 10, pp. 159-164, 2009.

[25] J. Guo, X. L. Chen, and H. Z. Jin, "Research on model of traffic flow based on cusp catastrophe," Control and Decision, vol. 23, no. 2, pp. 238-239, 2008.

[26] X. Ling, Catastrophe Theory and Application, Shanghai Jiaotong University Press, Shanghai, China, 1987.
[27] T. Chen and S. Chen, "Research on models of congestion control based on catastrophe theory," Journal of Systems Engineering, vol. 21, no. 6, pp. 598-605, 2007.

[28] G. Jian, X. Chen, and H. Jin, "Based on the cusp catastrophe toresearch the relationship among traffic flow three parameters," in Proceedings of the IEEE International Conference on Automation \& Logistics IEEE, pp. 858-861, Jinan, China, August 2007.

[29] Y. Wang, U. A. Weidmann, and H. Wang, "Using catastrophe theory to describe railway system safety and discuss system risk concept," Safety Science, vol. 91, pp. 269-285, 2017.

[30] J. Monteil, R. Billot, J. Sau, and N.-E. El Faouzi, "Linear and weakly nonlinear stability analyses of cooperative car-following models," IEEE Transactions on Intelligent Transportation Systems, vol. 15, no. 5, pp. 2001-2013, 2014.

[31] Y. Zhang and S. G. Li, "Analysis of scale-free characteristic on sharp variation point of traffic flow," Acta Physica Sinica, vol. 24, pp. 134-139, 2014.

[32] Y. Wang, Y. Zhang, X. Piao, H. Liu, and K. Zhang, "Traffic data reconstruction via adaptive spatial-temporal correlations," IEEE Transactions on Intelligent Transportation Systems, vol. 20, no. 4, pp. 1531-1543, 2019.

[33] H. Zhang, P. Chen, J. Zheng et al., "Missing data detection and imputation for urban ANPR system using an iterative tensor decomposition approach," Transportation Research Part C: Emerging Technologies, vol. 107, pp. 337-355, 2019.

[34] C. Lin, Y. Yu, D. Wu, and B. Gong, "Traffic flow catastrophe border identification for urban high-density area based on cusp catastrophe theory: a case study under sudden fire disaster," Applied Sciences, vol. 10, no. 9, p. 3197, 2020.

[35] Z. Li, H. Yu, G. Zhang, and J. Wang, "A Bayesian vector autoregression-based data analytics approach to enable irregularly-spaced mixed-frequency traffic collision data imputation with missing values," Transportation Research Part C: Emerging Technologies, vol. 108, pp. 302-319, 2019. 